\title{
EXTRAMUCOUS OESOPHAGOCARDIOMYOTOMY IN CARDIOSPASM
}

\author{
By RODNEY MAINGOT, F.R.C.S.Eng. \\ (Senior Surgeon, Southend General Hospital, and Royal Waterloo Hospital, \\ London; Consulting Surgeon, Essex County Council)
}

Cardiospasm or, as it is sometimes termed, achalasia of the oesophagus, is a condition in which there is dilatation and hypertrophy of the oesophagus associated with non-organic obstruction of the cardia.

The dysphagia is usually treated with antispasmodics such as amylnitrite or nitroglycerin combined with periodical dilatations of the cardia by. means of bougies, or more rarely with Plummer's hydrostatic bag. These measures will undoubtedly produce a symptomatic cure, or at least considerable amelioration, in many cases.

Operative measures should, however, be advised when:-

(I) Owing to the great size and sagging of the flask-which may be $\mathrm{J}$-shaped, dilatation even under direct vision is a hazardous undertaking or is not feasible.

(2) The bougies pass easily into the stomach, but even the largest size fails to relieve the dysphagia.

(3) The patient is un-coöperative or intolerant of the bougie method of management. strength.

(4) In spite of periodical dilatations there is a steady decline in the patient's weight and

In other words, the chief indication for operation is the failure of the dilatation method after this has been given a fair trial.

It is often erroneously considered that the various operations for cardiospasm are associated with a high mortality and a high recurrence rate. In spite of the fact that many patients with this condition are frail and undernourished, the approach to the cardia is difficult owing to its fixed position deep in the abdomen, and operative procedures upon the friable oesophagus are fraught with the dangers of sepsis, the all-round death-rate of all the various plastic operations does not exceed 9 per cent, and the failures are under ro per cent. Furthermore, by the method about to be described the percentage of recurrence and operative mortality is reduced to a bare minimum.

Ochsner and De Bakey concluded that the results were good in 80 per cent of all collected cases, and that improvement was observed in Io per cent. Heller (I9I3 and I92I) described I6 cases operated upon by him and other surgeons by extramucous oesophagocardiomyotomy without fatality and with good results in twelve cases, whilst Zaaiger (I923) gave a detailed account of eight further successful cases without mortality.

Recently, Barlow reported four cases treated successfully by his modification of the Heller type of oesophagocardiomyotomy, and since his publication in the British Journal of Surgery (I942) he has operated upon two additional cases which have done very well to date.

My small experience with the Mikulicz transgastric digital dilatation procedure has not been very encouraging, as in the three cases in which this operation was performed there was one death from mediastinitis, one failure after two operations, and only one case which was cured. This Mikulicz operation is, in many respects, similar to Loretta's method of divulsion of the fibres of the pyloric tumour in cases of infantile pyloric stenosis, but in cardiospasm the stretching of the cardia and of the oesophagus itself is performed blindly and by feel, and must therefore of necessity be uncertain in its results and not free from the dangers of splitting the gullet with the subsequent onset of mediastinitis.

Ochsner and De Bakey consider oesophagogastrostomy, the rationale of which is similar to Finney's gastroduodenostomy for stenosing duodenal ulcer, to be the operation of choice, and the published figures would appear to support their claim. Although the technical beauty, the ease of performance and the security that this operation affords against recurrence and complication $s$ do much to commend it, when compared with the other plastic procedures about the cardia it has nevertheless one flaw-regurgitation. In two of my cases regurgitation of gastric contents, especially when the patients were lying down, was a most distressing feature. Oesophagocardiomyotomy is, in my opinion, worthy of a more general adoption, as it is a simple and safe operation, the technique is readily mastered, and the immediate and late results are most gratifying. Furthermore, these statements are confirmed by the excellent results 
obtained in the four personal cases about to be described,* and in ten other cases which were successfully operated upon by this method and were reported to me by three British surgeons. In this small series of fourteen cases there were no deaths, and cures were obtained in all, although some of the operations are as yet of recent date.

\section{TECHNIQUE OF THE OPERATION}

The patient is admitted to hospital a few days before operation, and lavage of the oesophagus is carried out once or twice daily, using a weak sodium bicarbonate solution, until all signs of oesophagitis have disappeared. An oesophagoscope is then passed to ensure that the inflammatory process has subsided. The patient is given a well-balanced fluid diet rich in sugars and proteins and the essential vitamins. No solid food is allowed during the pre-operative phase.

About three hours before the operation is due to commence the oesophagus is thoroughly irrigated with warm normal saline solution, after which a Ryle tube is passed through the nostril into the stomach, and the gastric contents are aspirated. The Ryle tube is allowed to remain in situ during the operation and for the first two post-operative days.

Anaesthesia is induced with gas, oxygen and ether, or with pentothal sodium intravenously followed by cyclopropane.

The abdomen is opened through a left paramedian incision or a lengthy left oblique paracostal incision which starts at the midline just below the xiphisternum and finishes near the tip of the tenth rib. After tetra-cloths have been affixed to the skin margins and cellophane squares stitched to the cut edges of the peritoneum, the stomach is drawn firmly downwards, and the left lateral hepatic ligament is divided (Lambert, I9I3) in order to permit of ready retraction with a Deaver retractor of the left lobe of the liver and better exposure of the cardia.

The peritoneum over the oesophagus is incised for 2 inches or so at the site of its reflection on to the diaphram, and by blunt and sharp dissection the oesophagus is freed circumferentially. Several small branches of the left inferior phrenic and left suprarenal veins coursing across the oesophagus are ligated with fine silk and divided, after which the left vagus nerve is identified and drawn out of harm's way.

When the oseophagus has been freed from the diaphragmatic opening, a sling of gauze is placed around the gullet to permit of traction downwards on the oesophagus. This traction on the sling aids in the mobilisation of the oesophagus, which is gradually pulled down into the peritoneal cavity for approximately 3-4 inches.

A pad of gauze is next placed beneath the oesophagus and cardia, and a longitudinal incision fully 3-4 inches in length is made through the muscular coats of the oesophagus and cardiac region of the stomach, down to the mucosa. The incision is placed anteriorly and is made very carefully with a small knife through the longitudinal muscular coat and then through the thin somewhat adherent circular coat of the gullet, dividing the fibres until the oesophageal mucosa bulges outwards without restraint. When this is completed, it is an easy matter to prolong the incision through the cardia into the stomach wall until the much stouter gastric mucous membrane appears and expands in the newly-made furrow. Many small blood vessels lying on the mucous membrane of the oesophagus and cardia may need ligation with fine silk. In the average case the incision extends for 2 inches into the oesophagus and for $\mathrm{I} \frac{1}{2}$ inches through the cardiac region.

The operation is completed by grasping the body of the stomach and squeezing it to force air and gastric juice through the oesophagus to make quite sure that the thin exposed mucous membrane of the oesophagus and cardia has not been punctured. If the mucous membrane is accidentally nicked, the hole can be quickly closed with a series of closely-applied stitches of silk or catgut without fear of subsequent leakage.

The operation is based on the principle of Rammstedt's pyloromyotomy for infantile pyloric stenosis, but differs from this procedure in that there is no gritty tumour to incise, and there is no obstructing band or strictured area which can be seen with the naked eye.

An interesting feature in the results of this operation is the observation made by many surgeons that functionally the patients are considerably better than the X-ray pictures would suggest. Thus in many cases the gullet appears to be still dilated after the operation, although the patient is able to swallow normally. Barlow (I942), who makes a similar observation, states that "although swallowing remains normal, the radiological appearances may seem

* A further four cases have since been operated upon with equal succes-Personal communication. Ed. 
disappointing." Debet considers in this connection that the satisfaction of the radiographer is often less than that of the patient.

\section{Case Reports}

CASE I.-M.E., a married woman, aged 3I, a draper's assistant, was admitted to the Essex County Council Hospital, Wanstead, on 29.II.42. For two years she had had difficulty in taking her food. She said that the food stuck in her chest, and that sometimes it caused her to cough and vomit. Both liquid and solid foods alike produced dysphagia. She found that if she took small amounts of food slowly, she could get it down without undue difficulty. On occasion there was regurgitation of food, but she did not return any old food.

On radiography the barium swallow showed the typical picture of cardiospasm with marked delay in the entry of barium into the stomach.

Operation.-This was performed on 7.12.42 under endotracheal gas, oxygen and ether anaesthesia. The abdomen was opened through a left paracostal incision, and in this case there appeared to be a constricting band at the cardio-oesophageal junction which could be lifted up and displayed on an aneurysm needle. This was divided, and a long ( $3 \frac{1}{2}$-inch) incision was made through the muscular coats of the oesophagus and cardia down to the mucosa. There was a little trouble with bleeding from the oesophageal veins, but this was controlled with two or three silk ligatures.

The abdominal incision was closed with interrupted sutures of floss silk and deknatel.

Post-Operative Progress.- The patient was allowed sips of water on recovery from the anaesthetic. She was taking milk in 24 hours, and within 7 days she was on normal diet. She was sent home on the tenth day following operation, and since then has never complained of any difficulty in swallowing food.

One month later and again after two months the barium swallow X-ray examination showed no hold-up whatsoever in the passage of barium through the oesophagus to the stomach. She has put on weight, and has expressed herself as being entirely satisfied with the result of the operation.

CASE 2.-M.G., married woman, aged 6I, admitted to the Essex County Council Hospital, Wanstead, on I.I0.42. She stated that for ten years she had not been able to eat any solid food. In her own words: "Solid food would simply not go down." She had vomited on many occasions, and she salivated a great deal. Her symptoms had all been worse during the last three years, and she had been steadily losing weight. She had been treated elsewhere with mercury bougies, but she said that although this dilatation treatment did afford her some relief, she had been progressively going downhill and losing weight and strength in spite of always feeling hungry and thirsty.

On examination she was a wasted, feeble old woman, edentulous, with palpable cervical glands and bilateral inguinal herniae. Large faecal masses could be palpated in the ascending and descending colon.

A barium swallow X-ray examination showed gross dilatation and sagging of the oesophagus, and the appearance was characteristic of cardiospasm with delay in the passage of the opaque meal to the stomach.

Operation.-This was carried out on 5.10.42. The cardiac region was exposed through a left paracostal incision under gas, oxygen and ether anaesthesia.

The operation of extramucous oesophagocardiomyotomy was performed expeditiously and without event in twenty minutes.

Post-Operative Course.- The patient was given sulphadiazine, I grm. four-hourly for 4 days, as a prophylactic measure. She was allowed to take sips of water on recovery from the anaesthetic, and within 24 hours she was drinking sterile glucose water and diluted milk. This patient was very feeble, and the diet had to be stepped up to normal a little more slowly than usual. However, within a fortnight she was eating ordinary ward diet eagerly and with relish. She was discharged $2 \frac{1}{2}$ weeks after her operation, in good condition and with the wound soundly healed.

A barium swallow X-ray examination taken six weeks after the operation showed that there was still some dilatation of the oesophagus, but there was no delay in emptying, and there was no complaint of any difficulty in swallowing at any time after operation. The patient stated that she could not remember when she had felt so well, and that she could now eat a square meal for the first time in ten years. 
CASE 3.-F.L., married man, aged 56, commercial traveller, was admitted to the Essex County Council Hospital, Wanstead, on 20.6.43. He gave a history that for three years he had had pain in the left side and in the lower part of his chest after taking food. He also had a pain situated immediately behind the xiphisternum. He would vomit after food, and this would relieve the discomfort. He also found some alleviation from the taking of alkaline mixtures. There were intervals during which the discomfort was less, but he had not been entirely free from trouble for any length of time during these three years. His main complaint was that the food seemed to stick in the lower part of his chest.

On examination, he was a thin but relatively healthy-looking man. Barium swallow $\mathrm{X}$-ray examination showed the oesophagus to be dilated, there was delay in emptying, and the skiagrams gave the typical appearance of cardiospasm. On oesophagoscopy a diagnosis of cardiospasm or early cancer of the lower end of the oesophagus was made.

Operation was performed on 28.6.43. A left paramedian epigastric incision was made to explore the abdomen under endotracheal gas, oxygen and ether anaesthesia. In this case, whilst making an incision through the circular coat of the oesophagus, the mucous membrane was inadvertently incised. This little puncture hole was closed with interrupted sutures of fine silk.

Post-Operative Course.-Sulphathiazole, I grm. 4-hourly for 4 days; was given prophylactically. Sterile glucose water was prescribed for 24 hours, and after this he was given light diet until the seventh post-operative day, when he was allowed the ordinary hospital diet. At no time did the patient have any symptoms such as he had previously experienced on taking food.

Two months after operation barium swallow X-ray examination showed some change in the dilatation of the oesophagus, this being approximately half the previous size, although it still appeared to be dilated. The patient was, however, completely symptomless, and was taking his meals normally. He was examined again recently, when the oesophagus was found to be further reduced in size, and the patient had gained in weight and was taking ordinary diet.

CASE 4.-F.W., married woman, aged 53, was admitted to the Southend General Hospital on I3.7.43, complaining of regurgitation of food for over 30 years. For I5 years there had been a progressive difficulty in swallowing, with pain behind the lower part of the sternum, where the food seemed to stick. After food pain was sometimes intense, when she would make herself vomit by putting her fingers down her throat and thus relieve the pain. She often suffered from hiccoughs after food. All her symptoms were worse in attacks every two or three months, and during these attacks she was completely disabled.

Barium swallow X-ray examination showed obstruction of the lower end of the oesophagus with marked dilatation above. Oesophagoscopy failed to show any evidence of organic stricture.

Operation was undertaken on $\mathbf{7 7 . 7 . 4 3 . ~ A ~ l e f t ~ s u b c o s t a l ~ i n c i s i o n ~ w a s ~ m a d e ~ u n d e r ~ c o n t i n u o u s ~}$ spinal anaesthesia supplemented by cyclopropane. The left lobe of the liver was firmly fixed posteriorly and could not be mobilised. There was a considerable amount of indurated fat around the lower end of the oesophagus, which was freed with some difficulty. A rubber tube was passed around the lower end of the oesophagus and was used to apply traction and to steady the gullet. The oesophageal muscular coats were incised longitudinally over the lower 2 inches of the oesophagus and upper $I_{\frac{1}{2}}$ inch of the stomach. A good plane of cleavage was obtained between the longitudinal and circular muscular coats, but no cleavage of any account could be found between the circular muscular coat and the mucous membrane which was closely adherent to the muscle.

After incision, the mucous membrane protruded throughout the length of the incision, but was torn in one place where it had to be repaired with sutures of No. oo chromic catgut.

The abdominal incision was united, using continuous fine silk for the peritoneum, and interrupted floss silk sutures through all the muscular layers, the skin margins themselves being approximated with Michel clips.

Post-Operative Course.-For the first four post-operative days nothing was allowed by mouth, fluids being introduced by means of venoclysis. Thereafter the patient was given fluids, and on the tenth day a light diet. Her temperature showed slight pyrexia from the third to the ninth post-operative days rising to over $100^{\circ} \mathrm{F}$. She returned home fit and well on 6.8.43, taking full hospital diet without difficulty.

She was re-examined on 5.I0.43 by means of barium meal X-ray examination, and this showed that the oesophagus was normal in size, and that the emptying was rapid. She was able to take full diet without any difficulty, and her general health was excellent. 


\title{
- SUMMARY
}

(I) The main indication for operation in cases of cardiospasm is the failure of medical treatment and periodical dilatations.

(2) The operation of choice is extramucous oesophagocardiomyotomy, the technical details of which are given.

(3) Notes of four personal cases are recorded together with end-results of operation.

(4) The operation described is one of such technical simplicity that, in the writer's opinion, it should be more widely practised in the future for the intractable type of cardiospasm.

I should like to express my thanks to Dr. Desmond Irwin, M.B., B.Ch., the Medical Superintendent of the Essex County Council Hospital, Wanstead, for permission to publish the details of the first three cases above mentioned; to Mr. Albert Gild, F.R.C.S., for his assistance with these cases; and to Mr. A. K. Monro, F.R.C.S., Surgical Registrar to the Southend General Hospital, for operating successfully upon the fourth case, which was admitted to this hospital under my care.

\section{REFERENCES}

I) BARLOW, D., Brit. Jl.Surg. (I942), 29, 4 .

(2) OCHSNER, A. and DE BAKEY, M., Arch Surg. (1940), 41, 1146

(3) HELLER, E., Mitt. a.d. Grenzgeb d. Med. U. Chir. (I9I3), 27, 14I.

(4) HELLER, E., Verhandl. d. deut. Gesellsch f. chir. (I92I), 45, 144.

(5) ZAAIGER, J. H., Ann. Surg. (1923), 77, 615.

(6) LAMBERT, A. V.. S., Ann. Surg. (I913), 58, 415.

\section{RADIOLOGY IN DISEASES OF THE OESOPHAGUS}

\author{
By G. T. CALTHROP, M.D., (Cantab.), D.M.R.E., Camb. \\ (From the X-ray Department, Royal Free Hospital)
}

The radiological examination of the oesophagus can determine:-

I. The position of the oesophagus.

2. The size of its lumen.

3. The contour of the lumen.

4. The presence of any obstruction, its site and the degree.

The oesophagus is a tube whose walls, during the greater part of life, are collapsed, so that there is only a potential lumen. Consisting of muscular and connective tissue, it cannot be differentiated by X-rays from the surrounding tissues, but the lumen may be made visible by filling it with a substance which is of lesser or of greater density. Even if air, or other medium of lesser density could be used, it would be impossible to see details in the intra-thoracic portion because of the over-lying shadows and translucencies of the lungs and other structures. A medium of greater density, i.e. more opaque to X-rays, must be used, and a suspension of barium sulphate is now universally employed.

When it is required only to depict the course of the oesophagus, no previous preparation of the patient is necessary, but in all other cases no solids or even fluids should have been taken by mouth for at least four, and preferably more, hours before the examination.

The passage of the opaque medium through the oesophagus is observed in the usual manner on the fluoroscopic screen, and a radiographic record is made to permit study at leisure and a comparison in the future.

The demonstration of mucosal relief of the oesophagus can be made. Its value, as so often in the alimentary tract, is greatest when it is normal, and it gives greater confidence in the report that there is no abnormality. The details of the technique need not be described here. 